ORIGINAL ARTICLE

\author{
Robin Ruszat · Tullio Sulser • Michael Dickenmann \\ Thomas Wolff • Lorenz Gürke • Thomas Eugster \\ Igor Langer • Peter Vogelbach · Jürg Steiger \\ Thomas C. Gasser • Christian G. Stief \\ Alexander Bachmann

\section{Retroperitoneoscopic donor nephrectomy: donor outcome and complication rate in comparison with three different techniques}

Received: 18 October 2005/ Accepted: 30 December 2005/Published online: 25 January 2006

(C) Springer-Verlag 2006

\begin{abstract}
Four surgical techniques for living donor nephrectomy were analyzed retrospectively in terms of perioperative outcome and early complication rate. A total of 182 donor nephrectomies including 69 open (OLDN), 14 fully laparoscopic (LDN), 34 hand-assisted laparoscopic (HLDN) and 65 retroperitoneoscopic (RLDN) nephrectomies were analyzed. There was a significant difference in mean operating time (OPT) between the OLDN (160 min) and RLDN (150 min) as compared to the LDN (212 $\mathrm{min})$ and HLDN group $(192 \mathrm{~min})(P<0.001)$. Mean warm ischemia time (WIT) was significantly shorter with OLDN (114 s), RLDN $(121 \mathrm{~s})$ and HLDN (128 s) when compared to LDN $(238 \mathrm{~s})(P<0.001)$. Major complication rate was comparable among the groups. Independent of the preferred technique, donor nephrectomy is associated with complication rates. RLDN is comparable to OLDN in terms of OPT, WIT. Learning endoscopic donor nephrectomy could be associated with a higher complication rate.
\end{abstract}

Keywords Retroperitoneoscopy $\cdot$ Donor nephrectomy $\cdot$ Laparoscopy $\cdot$ Comparison of techniques

R. Ruszat $(\bowtie) \cdot$ T. Sulser · T. C. Gasser · A. Bachmann Department of Urology, University Hospital Basel, Spitalstr. 21, 4031 Basel, Switzerland

E-mail: rruszat@uhbs.ch

Tel.: +41-61-2657284

Fax: + 41-61-2657273

M. Dickenmann · J. Steiger

Division of Transplantation Immunology and Nephrology, University Hospital Basel, Basel, Switzerland

T. Wolff · L. Gürke · T. Eugster · I. Langer · P. Vogelbach Department of Surgery, University Hospital Basel, Basel, Switzerland

C. G. Stief · A. Bachmann

Department of Urology, Ludwig Maximilians University of Munich, Munich, Germany

\begin{abstract}
Abbreviations OLDN: Open living donor nephrectomy $\cdot$ HLDN: Hand-assisted laparoscopic living donor nephrectomy $\cdot$ RLDN:

Retroperitoneoscopic living donor nephrectomy - LDN: Laparoscopic living donor nephrectomy · OPT:

Operating time $\cdot$ WIT: Warm ischemia time
\end{abstract}

\section{Introduction}

Safety and efficiency of living donor nephrectomy are of utmost concern to the donor and the recipient. It is unique in that it affects a healthy volunteer rather than a sick patient. This makes it one of the most demanding surgical procedures. The surgical technique recommended must entail with the lowest possible morbidity without compromising the functional outcome of the graft.

During the last few years the use of minimally invasive procedures has been continuously increased because they seem to be superior to the open approach. Since Ratner et al. [9] first performed a laparoscopic living nephrectomy in 1995, it has become a widely accepted alternative for living donation. In order to facilitate certain technically challenging steps of endoscopic procedures, some surgeons prefer hand assistance [19]. Like open retroperitoneal access, retroperitoneoscopy implies a direct approach to retroperitoneal organs without interfering with intraperitoneal organs. Although this procedure is the most logical approach to the anatomic structures of the retroperitoneum, only few centers have reported a large number of kidney donations performed with a primary retroperitoneoscopic approach [15-17, 20].

In this study, we analyzed the perioperative donor outcome and early complication rate of retroperitoneoscopic living donor nephrectomy (RLDN) and compared it retrospectively to three surgical techniques of living donor nephrectomy. 


\section{Materials and methods}

From November 1997 to March 2004, 182 donor nephrectomies were performed at the Basel University Hospital. After using a standard open approach for donor nephrectomy (OLDN) for more than 10 years, laparoscopic donor nephrectomy (LDN) was introduced in 1997 and was performed up to the 14th case. After the 14th case, all laparoscopic procedures were performed with hand assistance (HLDN). After the 48th case, we switched to a retroperitoneoscopic technique, which has since become our favored approach for performing various renal surgical procedures. Multiple renal arteries, body mass index (BMI) $>30 \mathrm{~kg} / \mathrm{m}^{2}$ or previous extensive abdominal surgery were considered a contraindication to laparoscopic surgery. There was a total of five $(2.7 \%)$ endoscopic donations where conversion to open surgery was necessary. Finally, 177 consecutive living kidney donations were available for statistical analysis, including 69 OLDN, 12 LDN, 33 HLDN and 63 RLDN. Detailed donor characteristics are shown in Table 1. Rightsided nephrectomy was performed in $26.6 \% \quad(n=47)$ cases overall, including $n=26(37.7 \%)$ in the OLDN, $n=2(6.1 \%)$ in the HLDN and $n=19(30.2 \%)$ in the RLDN. Reasons for right-sided nephrectomy were multiple left-sided vessels $(n=24 ; 51 \%)$, right-sided renal artery stenosis $(n=6 ; 13 \%)$, upper renal pole artery $(n=5 ; 11 \%)$, early left-sided artery division $(n=8 ; 17 \%)$, right-sided arterial dysplasia $(n=2 ; 4 \%)$, venous anomalies $(n=1 ; 2 \%)$ and left-sided double pyelon $(n=1 ; 2 \%)$.

All potential donors were routinely evaluated according to a donation protocol and discussed in detail by the transplantation team consisting of nephrologists, urologists, visceral and vascular surgeons, transplantation coordinators, immunological laboratories and psychosomatic experts. Preoperatively, conventional or a contrast enhanced magnet resonance angiography was performed to evaluate the vascular anatomy in all donors. All intra- and postoperative complications within a period of 30 days were analyzed for this study.

A standard open extraperitoneal technique through a flank incision was used in OLDN. The laparoscopic approach was performed in a standard technique, as previously described [9]. Additionally, for hand assistance a commercial hand port device was placed into the periumbilical position for HLDN. Our detailed technique of RLDN has been published [15].
The technique of renal vessel transsection was different. In the OLDN, the renal vessels were transsected using scissors after placing Satinskýs clamps on the renal artery close to the aorta and the renal vein. During LDN, HLDN, and RLDN the renal artery and vein were transsected with a Multifire-Endo TA-30 2.5 vascular stapler (Autosuture ${ }^{\circledR}$ ).

Operating time (OPT) was defined as the period between skin incision and skin closure. We defined warm ischemia time (WIT) as the time from ligation of the renal artery to the time when clear outflow of the cold irrigation solution $\left(\right.$ Viaspan $\left.^{\circledR}\right)$ in the renal vein was detected. In order to minimize cold ischemia time, donation and implantation were performed simultaneously in adjacent operating rooms. After irrigation with approximately $200-300 \mathrm{ml}$ Viaspan ${ }^{\circledR}$, the harvested kidney was placed in a sterile plastic bag and taken to implantation immediately.

All data presented are expressed as mean \pm standard deviation or available cases (numbers), if suitable. Statistical analysis was performed using the SPSS 11.5 (SPSS Inc., Chicago, IL, USA) statistical software package. The Kruskal-Wallis- $H$ test was used to compare three or more groups of independent non-parametric numerical data. Each group was compared to the other by means of the Mann-Whitney $U$ test. The chisquare test was employed to compare categorical data. A $P$ value of less than 0.05 was considered to be statistically significant.

\section{Results}

The donor characteristics are presented in Table 1. Because a BMI $>30 \mathrm{~kg} / \mathrm{m}^{2}$ was a contraindication to laparoscopic nephrectomy, donors BMI in the LDN and HLDN group were statistically smaller than in the OLDN and RLDN groups $(P=0.007)$ (Table 1$)$. There was no significant difference in age among the groups $(P=0.06)$. OPT was significantly shorter with OLDN and RLDN, as compared to LDN or HLDN $(P<0.001)$ (Table 1). HLDN was approximately $20 \mathrm{~min}$ shorter than LDN $(212 \pm 34$ vs. $192 \pm 24$ min; $P=0.056)$. OPT was on average 10 min shorter with RLDN than with OLDN, but this difference did not reach statistical significance $(P=0.07)$. OPT with RLDN was on average 42.6 min shorter than with HLDN $(P<0.001)$. WIT was shorter with OLDN (114 $\pm 28 \mathrm{~s})$, HLDN (128 $\pm 52 \mathrm{~s})$
Table 1 Perioperative data and donor characteristics after 182 living kidney donations

\footnotetext{
Data rounded

${ }^{\text {a }}$ Statistical comparison of four groups was performed using Kruskal-Wallis $H$ test

b" Converted" excluded from statistical group comparison
}

\begin{tabular}{lllllll}
\hline & OLDN & LDN & HLDN & RLDN & Converted & $P^{\mathrm{a}, \mathrm{b}}$ \\
\hline Number & 69 & 12 & 33 & 63 & 5 & \\
Age (years) & $53 \pm 11$ & $46 \pm 10$ & $50 \pm 13$ & $53 \pm 10$ & $60 \pm 9$ & NS \\
BMI (kg/m ${ }^{2}$ ) & $26 \pm 3$ & $24 \pm 3$ & $24 \pm 3$ & $26 \pm 4$ & $26 \pm 3$ & 0.007 \\
Gender female/male & $45 / 24$ & $7 / 5$ & $27 / 6$ & $44 / 19$ & $1 / 4$ & NS \\
Side left/right & $43 / 26$ & $12 / 0$ & $31 / 2$ & $44 / 19$ & $4 / 1$ & $<0.001$ \\
Operating time (min) & $160 \pm 29$ & $212 \pm 34$ & $192 \pm 24$ & $150 \pm 46$ & $197 \pm 32$ & $<0.001$ \\
WIT (s) & $114 \pm 28$ & $238 \pm 69$ & $128 \pm 52$ & $121 \pm 39$ & $288 \pm 235$ & $<0.001$ \\
Blood loss (ml) & $210 \pm 133$ & $307 \pm 159$ & $208 \pm 232$ & $171 \pm 110$ & $825 \pm 434$ & 0.012 \\
Hospital stay (days) & $13 \pm 2$ & $13 \pm 3$ & $11 \pm 2$ & $11 \pm 4$ & $12 \pm 3$ & $<0.001$ \\
\hline
\end{tabular}


and $\operatorname{RLDN}(121 \pm 29 \mathrm{~s})$ than with $\operatorname{LDN}(238 \pm 69 \mathrm{~s})$ $(P<0.001)$. The difference of WIT between OLDN, HLDN and RLDN was not significant (Table 1) $(P=0.67)$. Mean blood loss was significantly higher with LDN $(307 \pm 159 \mathrm{ml})$ when compared to OLDN $(210 \pm 133 \mathrm{ml}), \quad$ HLDN $(208 \pm 232 \mathrm{ml})$ and RLDN $(171 \pm 110 \mathrm{ml})(P=0.012)$. Mean hospitalization time was significantly shorter after RLDN (10.7 \pm 3.9 days) and HLDN (11.2 \pm 2.1 days) compared to OLDN $(13.0 \pm 2.4$ days $)$ and $\operatorname{LDN}(12.6 \pm 3.1$ days $)(P<0.001)$ (Table 1). Detailed perioperative data of the five cases which required conversion to open surgery are listed in Table 1.

There was no significant difference in overall complication rate, when comparing the four groups within a period of 30 days postoperatively (Kruskal-Wallis $H$ test; $P=0.082$ ). Subanalysis of intraoperative, postoperative, major and minor complications revealed no significant differences, when comparing all groups together (Table 2). When comparing each group to the other, overall complication rate of LDN was statistically different to OLDN $(P=0.016), \operatorname{HLDN}(P=0.046)$ and RLDN $(P=0.017)$ (Table 3$)$. Incidence of major complications was similar in all groups (Table. 2, 3). Differences of minor, major, intra- or postoperative complications did not differ significantly between OLDN, HLDN and RLDN groups. Minor and major complications occurred as follows: 8 in the LDN group $(57.1 \%), 17$ in the OLDN group $(24.6 \%), 9$ in the HLDN group $(26.5 \%)$ and 16 in the RLDN group $(24.6 \%)$. Major complications included a pleural laceration requiring drainage $(n=1)$ and asthmatic distress $(n=1)$ in the OLDN group; conversions to open surgery because of visceral adhesions in an obese donor $(n=1)$ in the HLDN group; problems in freeing the kidney after a pyelonephritis $(n=1)$ and bleeding from a suprarenal vein $(n=1)$ led to conversion in the LDN group; conversion because of a renal artery disruption $(n=1)$ and because of two very short renal veins $(<1 \mathrm{~cm})$ on the right side, which opened directly into the vena cava and were only discovered intraoperatively $(n=1)$, ureteral injury $(n=1)$, chyloretroperitoneum with consecutive chylothorax $(n=1)$ and postoperative myocardial infarction in a 79-year-old female donor $(n=1)$ in the RLDN group (Table 2).

\section{Discussion}

The standard open extraperitoneal or transperitoneal access has proved to be a safe approach for donor nephrectomy during the last 50 years. It is associated with a low complication rate [8]. Most surgeons feel confident and familiar with this approach. However, there is no question that the incision can entail some very significant postoperative problems including prolonged postoperative pain, scarring, permanent flank bulge due to relaxation of the abdominal wall muscles, subcostal nerve injury or pleural irritation [3]. Correspondingly, minimal flank incision living donor nephrectomy was introduced as an alternative to the standard open approach [6]. It appears that the main argument for choosing this technique is to avoid the well-known learning curve associated with laparoscopic donor nephrectomy. Drawbacks of the minimal flank incision technique are a limited view of anatomical structures and limited working space which can render the handling of intraoperative problems very difficult.
Table 2 Perioperative complication rate within 30 days after open, fully laparoscopic, hand-assisted laparoscopic and retroperitoneoscopic approach for living donor nephrectomy $(n=182)$

Data presented as frequencies. Statistical comparison of four groups was performed using Kruskal-Wallis $H$ test

${ }^{a}$ Including converted cases

${ }^{\mathrm{b}}$ This case is part of the cases that had to be converted

${ }^{\mathrm{c}}$ Major complications related to severe disadvantages for the donors, including conversion, reoperation, transfusion or effects on graft function

\begin{tabular}{|c|c|c|c|c|c|}
\hline & OLDN & $\mathrm{LDN}$ & HLDN & RLDN & $P$ \\
\hline Number of cases & 69 & $14^{\mathrm{a}}$ & $34^{\mathrm{a}}$ & $65^{\mathrm{a}}$ & \\
\hline \multicolumn{6}{|l|}{ Intraoperative } \\
\hline Conversion to the open approach ${ }^{\mathrm{c}}$ & - & 2 & 1 & 2 & \\
\hline Pleura laceration with necessity of drainage ${ }^{c}$ & 1 & 0 & 0 & 0 & \\
\hline Bleeding with blood transfusion ${ }^{\mathrm{c}}$ & 0 & 0 & 1 & $1^{\mathrm{b}}$ & \\
\hline Ureter injury $^{\mathrm{c}}$ & 0 & 0 & 0 & 1 & \\
\hline Decapsulating upper renal pole & 0 & 0 & 2 & 0 & \\
\hline Total & $1(1.5 \%)$ & $2(14.3 \%)$ & $4(11.8 \%)$ & $4(6.2 \%)$ & 0.104 \\
\hline \multicolumn{6}{|l|}{ Postoperative } \\
\hline Severe wound pain & 4 & 1 & 1 & 1 & \\
\hline Hematoma requiring transfusion $^{c}$ & 0 & 0 & 0 & 1 & \\
\hline $\begin{array}{l}\text { Chyloretroperitoneum with } \\
\text { chylothorax requiring reoperation }^{\mathrm{c}}\end{array}$ & 0 & 0 & 0 & 1 & \\
\hline Paralysis, Emesis, Vomitus & 1 & 2 & 2 & 0 & \\
\hline $\begin{array}{l}\text { Wound infection/dehiscence/large } \\
\text { hematoma (no transfusion) }\end{array}$ & 4 & 3 & 1 & 1 & \\
\hline Transient fever within $24 \mathrm{~h}$ postop. & 0 & 0 & 1 & 1 & \\
\hline Pyelonephritis & 0 & 0 & 0 & 1 & \\
\hline Pneumonia & 2 & 0 & 0 & 2 & \\
\hline Pleural emphysema/effusion & 1 & 0 & 0 & 3 & \\
\hline Cardiac ischemia $^{\mathrm{c}}$ & 0 & 0 & 0 & 1 & \\
\hline Asthmatic distress ${ }^{\mathrm{c}}$ & 1 & 0 & 0 & 0 & \\
\hline Urinary problems (retention, prostatitis) & 3 & 0 & 0 & 1 & \\
\hline Total & $16(23.2 \%)$ & $6(42.9 \%)$ & $5(14.7 \%)$ & $13(20 \%)$ & 0.209 \\
\hline Major complication $^{\mathrm{c}}$ & $2(2.9 \%)$ & $2(14.3 \%)$ & $2(5.9 \%)$ & $7(10.7 \%)$ & 0.497 \\
\hline Minor complication & $15(21.7 \%)$ & $6(42.9 \%)$ & $7(20.6 \%)$ & $10(15.4 \%)$ & 0.152 \\
\hline
\end{tabular}


Table 3 Statistical comparison of intraoperative, postoperative, major and minor complications with LDN (learning period) in comparison to OLDN, HLDN and $\operatorname{RLDN}(n=182)$

\begin{tabular}{llllll}
\hline Technique & \multicolumn{2}{l}{ Type of complication } & & & \\
\cline { 2 - 6 } & Intraoperative & Postoperative & Major & Minor & Overall \\
\hline OLDN & 0.020 & 0.131 & 0.441 & 0.100 & 0.016 \\
HLDN & 0.812 & 0.037 & 0.871 & 0.118 & 0.046 \\
RLDN & 0.301 & 0.098 & 0.804 & 0.012 & 0.017 \\
\hline
\end{tabular}

Data presented as statistical probability using the Mann-Whitney test

Basic data obtained from Table 2

Since the early 1990s, laparoscopic techniques have been successfully adapted for various open urologic procedures, including laparoscopic living donor nephrectomy which was first described in 1995 [9]. Data is also available suggesting that it can increase the rate of kidney donations [12]. The laparoscopic access is technically challenging and is associated with longer OPT and WIT but it has been proven to lead to less perioperative pain, earlier mobilization and return to normal activities when compared to the open approach $[2,10,14,18]$.

But the great variety of techniques that are still competing with the standard open approach suggests that the optimal minimally-invasive alternative to OLDN has not yet been determined $[6,9,15,17,19]$. We started endoscopic living donor nephrectomy in 1997 using a full laparoscopic approach. The procedure was felt to be technically challenging so that hand assistance was introduced after the 14th case which seemed to greatly facilitate the procedure. Hand assistance is claimed to shorten the learning curve especially when starting laparoscopic surgery, and to minimize WIT and OPT $[2,14,16,18,19]$. The added surgeon's tactile sensation is believed to increase safety to a level comparable to open surgery. OPT of published series with HLDN is in a range of 177-294 $\min [2,14,18,19]$ but is certainly shorter than with LDN. On the other hand, OPT even in a very experienced center, was in excess of $200 \mathrm{~min}$ on average in a large series [5, 7]. Both figures are comparable to our data (Table 1).

The advantages of the retroperitoneoscopic technique compared to the OLDN have recently been described in detail by our group $[1,15]$. Blunt and quick creation of the retroperitoneal space obviates the time-consuming adhesiolysis, and the retraction of the bowel or omentum, which especially in obese donors can be very cumbersome. Additionally, the medial fixation of the kidney with the Gerota's fascia and establishment of a pneumoretroperitoneum means that the kidney is lifted up and the renal vessels are tautened out in front of the camera leading to an optimal exposure during retroperitoneoscopic dissection. No peritoneal dissection or bowel retraction is necessary.

Several variations of RLDN have been described in order to optimize the procedure [16, 17].

The present study shows that OPT and WIT with RLDN are not significantly longer when compared to OLDN (Table 1). In addition, OPT with RLDN was significantly shorter than with HLDN and LDN $(P<0.001)$. Also, other authors have noted that the retroperitoneoscopic approach tended to be slightly shorter than open surgery $[17,20]$. In all the published series, OPT using a retroperitoneoscopic approach was very similar within a range of 130-158 min [1, 17, 20]. Some authors have experienced a dramatic shortening of OPT after having introduced hand assistance for retroperitoneoscopic donor nephrectomy from 238 to $166 \min$ [16]. However, duration of operation is not the most important issue to point out, but it indirectly gives us information about the feasibility, level of difficulty and reproducibility of a surgical technique.

Warm ischemia time with LDN or HLDN has been reported to be significantly longer than during OLDN. It is important to note that very different definitions of WIT are being used, so that the WIT between different studies can hardly be compared. But there is no doubt that vessel transsection in endoscopic procedures is technically more demanding despite various tactical improvements than during the open approach. Our data shows that WIT after RLDN or HLDN is not necessarily longer than with OLDN.

When discussing advantages or disadvantages of various techniques for donor nephrectomy it has to be pointed out, that it has been shown that graft function and the recipient's overall complication rate after transplantation of a kidney harvested by laparoscopic or retroperitoneoscopic donor nephrectomy are equal than after open nephrectomy [1, 11, 17, 19].

The overall rate of complications including minor and major complications of the present series was statistically similar among the groups (Table 2). The present study thus confirms that endoscopic donor nephrectomy has a low complication rate, regardless of the preferred technique, if performed by surgeons skilled in laparoscopic surgery. "Major complications" included events such as blood transfusions, reinterventions or conversion to open surgeries even though none of these events led to persistent disadvantages in the donor. The major complication rate in all four different techniques used in our series is comparable with published data: $1-6$ and 1$6.3 \%$ for OLDN and LDN/HLDN, respectively $[4,5,10$, 14]. A higher intra- $(6.8 \%)$ and postoperative $(17.1 \%)$ major complication rate in a large series of LDN has been reported [7]. Nevertheless, the rather high overall complication rate in our LDN group must be attributed 
to our learning curve. It depends on the definition of complication and the small sample size and can, therefore, not really be compared with the results achieved in other centers, using this technique for years $[4,5,10]$. Thus, the LDN group reflects our initial experience with endoscopic donor nephrectomy. However, mainly minor complications have occurred with LDN that will not have any impact on the donor's long-term well-being (Table 3). It has to be pointed out, that complication rate is increased during the learning period of endoscopic techniques for donor nephrectomy and introducing hand assistance might be a suitable tool to improve safety and feasibility during the learning period.

Additionally, the present study suggests that the type of complication might differ depending on the technique used (Table 2): OLDN was associated with a low intraoperative complication rate $(1.5 \%)$, but tends to entail more postoperative wound problems $(n=8$; $11.6 \%)$. LDN/HLDN tended to cause more bowel problems $(n=5 ; 16.3 \%)$ whereas RLDN seemed to be associated with more pleural and pulmonal complications $(n=4 ; 6.3 \%)$ (Table 2$)$. It must be pointed out that pleural complications are not exclusive of retroperitoneoscopy, as pneumomediastineum and atelectasis have been reported at a rate of $5.6 \%$ with open donor nephrectomy too [13]. For RLDN, as a result of our experience, we try to place the first $12 \mathrm{~mm}$ trocar more distally than the tip of the 12th rib in order to avoid these complications.

\section{Conclusion}

Retroperitoneoscopic donor nephrectomy was comparable to open donor nephrectomy in terms of OPT and WIT, while offering endoscopic features. Donors operated by an RLDN or HLDN were superior in terms of hospital stay when compared to the standard open approach. The overall complication rate was statistically similar independent of the technique used, although open donor nephrectomy tended to entail more postoperative complications whereas endoscopic procedures tended to be associated with more intraoperative problems. Full laparoscopic donor nephrectomy is associated with a higher complication rate during the learning period. The retroperitoneoscopic approach enables an easy technique, is characterized by optimal exposure during vessel dissection, and provides reproducible results in terms of short OPT and WIT while being associated with a low complication rate, which is comparable to the standard open donor nephrectomy.

\section{References}

1. Bachmann A, Dickenmann M, Gurke L, Giannini O, Langer I, Gasser TC, Steiger J, Sulser T (2004) Retroperitoneoscopic living donor nephrectomy: a retrospective comparison to the open approach. Transplantation 78:168-171
2. Buell JF, Hanaway MJ, Potter SR, Cronin DC, Yoshida A, Munda R, Alexander JW, Newell KA, Bruce DS, Woodle ES (2002) Hand-assisted laparoscopic living-donor nephrectomy as an alternative to traditional laparoscopic living-donor nephrectomy. Am J Transplant 2:983-988

3. Chatterjee S, Nam R, Fleshner N, Klotz L (2004) Permanent flank bulge is a consequence of flank incision for radical nephrectomy in one half of patients. Urol Oncol 22:36-39

4. Flowers JL, Jacobs S, Cho E, Morton A, Rosenberger WF, Evans D, Imbembo AL, Bartlett ST (1997) Comparison of open and laparoscopic live donor nephrectomy. Ann Surg 226:483-489

5. Giessing M, Deger S, Ebeling V, Roigas J, Turk I, Loening SA (2003) Die laparoskopische transperitoneale Donornephrektomie. Urologe A 42:218-224

6. Greenstein MA, Harkaway R, Badosa F, Ginsberg P, Yang SL (2003) Minimal incision living donor nephrectomy compared to the hand-assisted laparoscopic living donor nephrectomy. World J Urol 20:356-359

7. Jacobs SC, Cho E, Foster C, Liao P, Bartlett ST (2004) Laparoscopic donor nephrectomy: the University of Maryland 6years experience. J Urol 171:47-51

8. Johnson EM, Remucal MJ, Gillingham KJ, Dahms RA, Najarian JS, Matas AJ (1997) Complications and risk of living donor nephrectomy. Transplantation 64:1124-1128

9. Ratner LE, Ciseck LJ, Moore RG, Cigarroa FG, Kaufman HS, Kavoussi LR (1995) Laparoscopic live donor nephrectomy. Transplantation 60:1047-1049

10. Ratner LE, Kavoussi LR, Sroka M, Hiller J, Weber R, Schulam PG, Montgomery R (1997) Laparoscopic assisted live donor nephrectomy - a comparison with the open approach. Transplantation 63:229-233

11. Ratner LE, Montgomery RA, Maley WR, Cohen C, Burdick J, Chavin KD, Kittur DS, Colombani P, Klein A, Kraus ES, Kavoussi LR (2000) Laparoscopic live donor nephrectomy: the recipient. Transplantation 69:2319-2323

12. Schweitzer EJ, Wilson J, Jacobs S, Machan CH, Philosophe B, Farney A, Colonna J, Jarrell BE, Bartlett ST (2000) Increased rates of donation with laparoscopic donor nephrectomy. Ann Surg 232:392-400

13. Spanos PK, Simmons RL, Lampe E, Rattazzi LC, Kjellstrand CM, Goetz FC, Najarian JS (1974) Complications of related kidney donation. Surgery 76:741-747

14. Stifelman MD, Hull D, Sosa RE, Su L, Hyman M, Stubenbord W, Shichman S (2001) Hand assisted laparoscopic donor nephrectomy: a comparison with the open approach. J Urol $166: 444-448$

15. Sulser T, Gurke L, Langer I, Dickenmann M, Steiger J, Gasser TC, Bachmann A (2004) Retroperitoneoscopic living donor nephrectomy: first clinical experiences after 19 operations. J Endourol 18:257-262

16. Sundqvist P, Feuk U, Haggman M, Persson AE, Stridsberg M, Wadstrom J (2004) Hand-assisted retroperitoneoscopic live donor nephrectomy in comparison to open and laparoscopic procedures: a prospective study on donor morbidity and kidney function. Transplantation 78:147-153

17. Suzuki K, Ishikawa A, Ushiyama T, Fujita K (2002) Retroperitoneoscopic living donor-nephrectomy without gas insufflation: five years Hamamatsu University Experience. Transplant Proc 34:720-721

18. Velidedeoglu E, Williams N, Brayman KL, Desai NM, Campos L, Palanjian M, Wocjik M, Bloom R, Grossman RA, Mange K, Barker CF, Naji A, Markmann JF (2002) Comparison of open, laparoscopic, and hand-assisted approaches to live-donor nephrectomy. Transplantation 74:169-172

19. Wolf JS Jr, Marcovich R, Merion JW, Konnak RM (2000) Prospective, case matched comparison of hand assisted laparoscopic and open surgical live donor nephrectomy. J Urol 163:1650-1653

20. Yang SC, Ko WJ, Byun YJ, Rha KH (2001) Retroperitoneoscopy assisted live donor nephrectomy: the Yonsei experience. J Urol 165:1099-1102 